\title{
Antimalarial Drug Resistance: Surveillance and Molecular Methods for National Malaria Control Programmes
}

\author{
Umberto D'Alessandro
}

Prince Leopold Institute of Tropical Medicine, Antwerp, Belgium

\begin{abstract}
National malaria control programmes have the responsibility to develop a policy for malaria disease management based on a set of defined criteria as efficacy, side effects, costs and compliance. These will fluctuate over time and national guidelines will require periodic re-assessment and revision. Changing a drug policy is a major undertaking that can take several years before being fully operational. The standard methods on which a decision can be taken are the in vivo and the in vitro tests. The latter allow a quantitative measurement of the drug response and the assessment of several drugs at once. However, in terms of drug policy change its results might be difficult to interpret although they may be used as an early warning system for 2 nd or $3 r d$ line drugs. The new WHO 14-days in vivo test addresses mainly the problem of treatment failure and of haematological parameters changes in sick children. It gives valuable information on whether a drug still 'works'. None of these methods are well suited for large-scale studies. Molecular methods based on detection of mutations in parasite molecules targeted by antimalarial drugs could be attractive tools for surveillance. However, their relationship with in vivo test results needs to be established.
\end{abstract}

Key words: antimalarial drug resistance - in vivo test - in vitro test - polymerase chain reaction - surveillance

Despite considerable efforts done during this century to eradicate or control it, Plamodium falciparum malaria is still the most prevalent and the most devastating disease in the tropics (WHO 1993). In the last decades, its control and treatment has been complicated by the emergence of resistance to widely used antimalarial drugs such as chloroquine. Drug resistance of malaria parasites has been defined as the ability of a parasite strain to multiply or to survive in the presence of concentrations of a drug that normally destroy parasites of the same species or prevent their multiplication. Its dynamics and occurrence are the result of several interactions:

Parasites and drugs - Natural populations of P. falciparum are heterogeneous mixtures of individuals with different, genetically determined degrees of drug response. The efficacy of medication will depend on the concentration of the drug in relation to the parasite's sensitivity and the time over which concentrations above this threshold are maintained. A small fraction of the original parasite population might always survive to the drug but it will be eventually removed by the immune system. However, the infection will not be cleared if the surviving fraction is too large due either to

Fax: +32-3-247.6362. E-mail: UDAlessandro@proto.itg.be Received 15 June 1998

Accepted 30 July 1998 reduced sensitivity or to subcritical drug concentrations. Selection of resistant strains could occur when a particular drug is misused (Wernsdorfer 1991). The transmission of such parasites might also be enhanced by an increased production of gametocytes (Robert et al. 1996).

Humans and drugs - Readily absorbed drugs with a long half-life, like mefloquine and sulfadoxine-pyrimethamine (SP), can permit effective single dose treatment of malaria and the following chemoprophylactic period prevents infection for several weeks and may be important in recovery from anaemia. However, these drugs are likely to exert undesirable drug pressure for a long time once their concentrations drop below the critical threshold and may select resistant parasites. This has been shown in Kenya where a potent selective pressure for resistance operates even under conditions of supervised drug administration and optimal dosage (Watkins et al. 1997).

Vector and parasite - Vectors may be more receptive to resistant strains and may produce more parasites compared to sensitive strains. Enhanced drug pressure and uninhibited transmission might produce a fast selection and spread of resistant parasites (Wernsdorfer 1991).

\section{NATIONAL DRUG POLICIES}

It is the responsibility of National Programmes to develop a policy for malaria disease management as early diagnosis and adequate treatment remain the basic elements of any malaria control action 
(WHO 1993). The basis for the national drug policy should be a set of defined criteria for efficacy, side effects, costs and compliance. Rational prescribing in the public sector should be promoted through the development and introduction of treatment guidelines. These activities should be supported by the supply system to ensure that the drugs health workers have been trained to prescribe are actually available (Baudon 1995). Many of the factors influencing the national drug policy, such as parasite drug susceptibility, drug pricing and availability, will fluctuate over time and national guidelines for malaria treatment will require periodic re-assessment and revision (WHO 1996). This should be done on the basis of reliable information that, in case of parasite drug susceptibility should be collected by means of an appropriate surveillance system.

Implementing a change in drug policy is a major undertaking that can take several years before being fully operational. The 'reaction-time' could be quite long in countries where the capabilities of training and re-training of health workers is limited and the distribution system of drugs is complex, fragmentary and uncontrolled. A study done in Congo in 1993 has shown that 77\% of General Practitioners (GPs) did not know the national drug policy and that they continued to apply a strategy (weekly malaria chemoprophylaxis in children under 5) abandoned seven years before (Baudon 1995). In optimal conditions the reaction time has been estimated to at least two years (Baudon 1995). Considering these difficulties, once the decision of changing the first line drug has been taken there is no go back.

\section{SURVEILLANCE}

One of the major questions is whether the firstline drug is still 'working'. Unfortunately, the criteria on which this decision can be taken are not clear (Bloland et al. 1993). The standard methods to assess the efficacy of a given drug can be divided in two broad groups: in vivo and in vitro tests.

In vivo test - During the in vivo test the recommended dose of an antimalarial drug is administered to infected subjects and the parasite's response in the host is assessed. The test could be done on symptomatic or asymptomatic people (Wernsdorfer \& Payne 1988) with a 7-day followup (Prasad et al. 1990). It assesses only the initial parasitological response and, to a limited extent, clinical response to therapy during the follow-up. However, it does not address the implications and manifestations of persistent parasitaemia occurring after poor response, for example its impact on other condition such as anemia and malnutrition (Bloland et al. 1993). A study carried out in Malawi and in Kenya among young children with clinical malaria showed a shortening of the duration of clinical improvement and a decreased haematological recovery after therapy with chloroquine as compared to SP. In addition the health care system is burdened by both the drug cost and the manpower requirements of frequent and repetitive visits for treatment with a poorly efficacious drug. The new 14-days in vivo test proposed by WHO (WHO 1996) tries to address this problem by looking mainly at treatment failure and change in the haematological parameters $(\mathrm{Hb} / \mathrm{PCV}$ at day 0 and 14). It is a more clinical test, carried out on sick children (fever + parasitaemia) aged 6 months-5 years (D'Alessandro et al. 1997). This test does not permit a quantitative assessment of the drug sensitivity of individual parasite populations, may occasionally be influenced by the abnormal fate of the drug in individual patients and it is influenced by the immunological host response to the parasite. However, it gives an information that is closer to real-life situation and therefore essential in deciding drug policy changes.

In vitro test - The in vitro test consists in measuring the inhibition of schizont maturation by increasing doses of a given antimalarial drug. It allows the quantitative measurement of drug response, permits to test several drugs at once and imitate the non-immune state. However, it is generally held that the in vitro tests do not reflect the degree of in vivo resistance, since the latter is substantially determined by factors related to the host's response (Draper et al. 1988). Furthermore, it is estimated that the technical capacity to conduct the necessary assays is difficult to develop and maintain in national malaria control programmes. Therefore, in vitro testing cannot substitute in vivo observations of malaria therapy and is inappropriate for making policy decision on drug use. Nevertheless, in vitro tests may provide an early warning of impeding resistance before this becomes clinically apparent. The optimal deployment of in vitro tests should be to define specific issues related to temporal and geographical trends of parasite's response to drugs. Such issues include the longitudinal follow-up of parasite drug susceptibility, monitoring the patterns of parasite cross-resistance to different drugs and the establishment of baseline data on the susceptibility of local parasites to new drugs (WHO 1990).

\section{MOLECULAR METHODS FOR SURVEILLANCE}

Recently, molecular diagnostic methods for detecting resistant parasites have been proposed for monitoring the level and spread of resistance (Plowe et al. 1995). The methods are suited for use on large numbers of samples in a laboratory in a malaria-endemic country and have major advan- 
tages on in vitro tests that require parasite cultivation and take days to perform (Plowe et al. 1996). These molecular tools are based on the detection by PCR of point mutations in the parasite genes responsible for in vitro resistance. Presently, several mutations associated with resistance to SP have been identified while the role of those linked to chloroquine resistance is more controversial. The latter has been associated to Pfmdrl gene polymorphysm, although multiple mutations in different genes are probably required for clinical resistance (Foote et al. 1990). Wellems et al. (1990) have linked chloroquine resistance to a single genetic locus yet to be identified. The point mutations linked to SP resistance have been observed in the parasite genes encoding for dihydrofolate reductase (DHFR) and dihydropteroate synthetase (DHPS), the targets for pyrimethamine and sulfadoxine respectively (Wang et al. 1995). A serine in position 108 of the DHFR gene is linked to in vitro sensitivity to both pyrimethamine and cycloguanil. A mutation to asparigine at position 108 seems to be the key mutation for conferring in vitro pyrimethamine resistance (de Pecoulas et al. 1996), although a genotype without this mutation has been recently described (Wang et al. 1997). An asparagine to isoleucine change at position 51 and a cysteine to arginine at position 59 appear to modulate higher levels of in vitro pyrimethamine resistance when they occur with the asparagine108 mutation, and an isoleucine to leucine mutation at position 164 in combination with the asparagine-108 and arginine-59 mutations has been found in $P$. falciparum lines that are highly resistant to both pyrimethamine and cycloguanil (Basco et al. 1995, Reeder et al. 1996). Point mutations of the DHPS gene have been less extensively studied. Thirteen variants over the wild type have been identified so far in samples from different countries, the most common alteration being in position 437 (Wang et al. 1997). A certain amount of correlation was found between the prevalence of known DHFR and DHPS mutations and increasing levels of in vivo SP resistance in four different countries: Mali, Kenya, Malawi, Bolivia (Plowe et al. 1997). Different degrees of in vivo parasitological resistance and clinical failure to SP might be due to the progressive accumulation of DHFR and DHPS mutations (Plowe et al. 1997). However, this still needs to be established by carrying out prospective studies on the relationship between parasite genotype and clinical outcome in individual infections treated with SP. No relation between clinical SP resistance and mutations in the DHFR gene could be established in a study carried out in Tanzania. Although all the isolates showed a point mutation in at least one codon of the DHFR gene, only $43 \%$ of the children had detectable parasitaemia seven days after treatment (Jelinek et al. 1997).

The characterisation of the two polymorphic merozoite surface antigens, MSP1 and MSP2 has also been used to establish whether a parasitaemia observed after treatment is caused by a recrudescence of drug-resistant parasites or by a new infection (Babiker et al. 1994, Al-Yaman et al. 1997). This could be an important information when carrying out in vivo tests, particularly in areas with a considerable amount of transmission where, after a certain time, it is impossible to distinguish between recrudescence and new infection. However, it is unlikely that national malaria control programmes will routinely use such methods.

In vitro test results have been used as the golden standard to establish the link between resistance to SP and point mutations in the DHFR and DHPS genes. Therefore, molecular tests identifying mutant parasites might replace in vitro tests, as they are easier to perform and can be carried out on a larger number of samples. They will complement the information on treatment failure and might actually identify areas where a given drug is likely to be less efficacious. However, for antimalarial drugs other than SP, these tests are not available yet and drug susceptibility of different parasite populations will continue to be established by in vitro tests. Decisions on the national drug policy will continue to be based on the results of in vivo tests as these reflect more closely the therapeutic efficacy of a given drug.

\section{REFERENCES}

Al-Yaman F, Genton B, Reeder JC, Anders RF, Alpers MP 1997. Evidence that recurrent Plasmodium falciparum infection is caused by recrudescence of resistant parasites. Am J Trop Med Hyg 56: 436-439.

Babiker H, Randford-Cartwright L, Sultan A, Satti G, Walliker D 1994. Genetic evidence that RI choloroquine resistance of Plasmodium falciparum is caused by recrudescence of resistant parasites. Trans $R$ Soc Trop Med Hyg 88: 328-331.

Basco LK, de Pecoulas PE, Wilson CM, Le Bras J, Mazabraud A 1995. Point mutations in the dihydrofolate reductase-thymidylate synthase gene and pyrimethamine and cycloguanil resistance in Plasmodium falciparum. Mol Biochem Parasitol 69: 135-138.

Baudon D 1995. La prise de décision dans la politique d'utilisation des antipaludiques en réponse à une modification de l'efficacité de la chloroquine. Réflexions appliquées à l'Afrique. Méd Trop 55: 37S-40S.

Bloland PB, Lackritz EM, Kazembe PN, Were JBO, Steketee R, Campbell CC 1993. Beyond chloroquine: implications of drug resistance for evaluating malaria therapy efficacy and treatment policy in Af- 
rica. J Infect Dis 167: 932-937.

D’Alessandro U, Mutabingwa TK, Ouma JH, Watkins WM 1997. Report of the workshop on monitoring antimalarial treatment efficacy: an East African regional network. Malaria Consortium, UK.

De Pecoulas PE, Basco LK, Le Bras J, Mazabraud A 1996. Association between antifol resistance in vitro and DHFR gene point mutation in Plasmodium falciparum isolates. Trans $R$ Soc Trop Med Hyg 90: 181-182.

Draper CC, Hills M, Kilimali VAEB, Brubaker G 1988. Serial studies on the evolution of drug resistance in malaria in an area of East Africa: findings from 1979 up to 1986. J Trop Med Hyg 91: 265-273.

Foote SJ, Kyle DE, Martin RK, Oduola AM, Forsyth K, Kemp DJ, Cowman AF 1990. Several alleles of the multidrug-resistance gene are closely linked to chloroquine resistance in Plasmodium falciparum. $\mathrm{Na}$ ture 345: 255-258.

Jelinek T, Ronn AM, Curtis J, Duraisingh MT, Lemnge MM, Minha J, Bygbjerg IC, Warhurst DC 1997. High prevalence of mutations in the dihydrofolate reductase gene of Plasmodium falciparum in isolates from Tanzania without evidence of an association to clinical sulfadoxine/pyrimethamine resistance. Trop Med Intern Hlth 2:1075-1079.

Plowe CV, Djimde A, Bouare M, Doumbo O, Wellens TE 1995. Pyrimethamine and proguanil resistanceconferring mutations in Plasmodium falciparum dihydrofolate reductase: polymerase chain reaction methods for surveillance in Africa. Am J Trop Med Hyg 52: 565-568.

Plowe CV Djimde A, Wellems TE, Diop S, Kouriba B, Doumbo OK 1996. Community pyrimethaminesulfadoxine use and prevalence of resistant Plasmodium falciparum genotypes in Mali: a model for deterring resistance. Am J Trop Med Hyg 55: $467-$ 471.

Plowe CV, Cortese JF, Djimde A, Nwanyanwu OC, Watkins WM, Winstanley PA, Estrada-Franco JG, Mollinedo RE, Avila JC, Cespedes JL, Carter D, Doumbo OK 1997. Mutations in Plasmodium falciparum dihydrofolate reductase and dihydropteroate synthase and epidemiological patterns of pyrimethamine-sulfadoxine use and resistance. J Infect Dis 176: 1590-1596.

Prasad RN, Prasad H, Virk KJ, Sharma VP 1990. Application of a simplified in-vivo test system for determining chloroquine resistance in Plasmodium falciparum. Bulletin WHO 68: 755-758.

Reeder JC, Rieckmann KH, Genton B, Lorry K, Wines B, Cowman AF 1996. Point mutations in the dihydrofolate reductase and dihydropteroate synthetase genes and in vitro susceptibility to pyrimethamine and cycloguanil of Plasmodium falciparum isolates from Papua New Guinea. Am J Trop Med Hyg 55: 209-213.

Robert V, Molez JF, Trape JF 1996. Short report: gametocytes, chloroquine pressure, and the relative parasite survival advantage of resistant strains of falciparum malaria in West Africa. Amer J Trop Med Hyg 55: 350-351.

Wang P, Brooks DR, Sims PFG, Hyde JE 1995. A mutation-specific PCR system to detect sequence variation in the dihydropteroate synthetase gene of Plasmodium falciparum. Mol Biochem Parasitol 71: 115125.

Wang P, Lee CS, Bayoumi R, Djimde A, Doumbo O, Swedberg G, Dao LD, Mshinda H, Tanner M, Watkins WM, Sims PFG, Hyde JE 1997. Resistance to antifolates in Plasmodium falciparum monitored by sequence analysis of dihydropteroate synthetase and dihydrofolate reductase alleles in a large number of field samples of diverse origins. Mol Biochem Parasitol 89: 161-177.

Watkins WM, Mberu EK, Winstanley PA, Plowe CV 1997. The efficacy of antifolate antimalarial combinations in Africa: a predictive model based on pharmacodynamic and pharmacokinetic analyses. Parasitol Today 13: 459-464.

Wellems TE, Panton LJ, Gluzman IY, do Rosario VE, Gwadz RW, Walker-Jonah A, Krogstad DJ 1990. Chloroquine resistance not linked to $m d r$-like genes in a Plasmodium falciparum cross. Nature 345: 253255.

Wernsdorfer WH, Payne D 1988. Drug sensitivity tests in malaria parasites, p. 1765-1794. In WH Wernsdorfer, I McGregor (eds), Malaria - Principles and Practice of Malariology, Churchill Livingstone, Edinbourg, London, Melbourn, New York.

Wernsdorfer WH 1991. The development and spread of drug-resistant malaria. Parasitol Today 11: 297-303.

WHO - World Health Organization 1990. Practical chemotherapy of malaria. Technical Report Series 805. WHO, Geneva.

WHO - World Health Organization 1993. Implementation of the global malaria control strategy. Report of a WHO Study Group on the implementation of the Global Plan of Action for Malaria Control 19932000. WHO, Geneva.

WHO - World Health Organization 1996. Assessment of therapeutic efficacy of antimalarial drugs for uncomplicated falciparum malaria in areas with intense transmission. WHO/MAL/96. 1077. 